05

\title{
Зарождение и распространение температурного фронта при кристаллизации аморфного сплава $\mathrm{Ti}_{50} \mathrm{Cu}_{50}$
}

\author{
(C) Е.Е. Слядников, ${ }^{1,2}$ Ю.А. Хон, ${ }^{1}$ П.П. Каминский, ${ }^{1}$ И.Ю. Турчановский ${ }^{2}$ \\ ${ }^{1}$ Институт фозики прочности и материаловедения СО РАН, \\ 634055 Томск, Россия \\ ${ }^{2}$ Институт вычислительных технологий СО РАН, \\ 630090 Новосибирск, Россия \\ e-mail: nadiet@mail.ru
}

(Поступило в Редакцию 24 мая 2017 г. В окончательной редакции 23 июля 2017 г.)

\begin{abstract}
Построена кинетическая модель, выявлены физические причины, условия, механизм и закономерности зарождения и распространения температурного фронта при термоактивированном аморфно-кристаллическом превращении в $\mathrm{Ti}_{50} \mathrm{Cu}_{50}$, инициированном объемным тепловым источником. Эта модель количественно и качественно описывает результаты и закономерности распространения температурного фронта, полученные экспериментально.
\end{abstract}

DOI: $10.21883 /$ JTF.2018.03.45593.2347

\section{Введение}

Аморфные сплавы металлов привлекают большое внимание исследователей благодаря уникальным механическим, электромагнитным, теплофизическим и антикоррозионным свойствам, что делает их перспективными материалами для современных технологий. Совсем недавно были обнаружены и экспериментально изучены самораспространяющиеся волны кристаллизации в предварительно нагретом постоянным электрическим током аморфном сплаве $\mathrm{Ti}_{50} \mathrm{Cu}_{50}$ [1]. Анализ последовательности температурных профилей самораспространяющихся волн в режимах самопроизвольного и инициированного локальным поверхностным тепловым источником превращения позволяет заключить, что они обладают рядом особенностей. Во-первых, зарождение и распространение волн существенно зависит от температуры образца. Во-вторых, переход из аморфного состояния в кристаллическое состояние происходит без плавления. В-третьих, фазовый состав системы при указанном превращении не меняется.

Механизм самораспространяющихся волн в металлических стеклах остается не ясным. С одной стороны, зависимость зарождения и распространения волн от температуры нагрева образца свидетельствует об их тепловой природе. С другой стороны, разность (перепад) температур между аморфным (перед фронтом волны) и кристаллическим (за фронтом) состояниями образца составляет $\approx 150^{\circ} \mathrm{C}[1]$. Поэтому высокая подвижность атомов во фронте самораспространяющейся волны не может быть объяснена наличием короткоживущего слоя расплава во фронте кристаллизации, поскольку температура за фронтом волны значительно ниже температуры плавления интерметаллида. Переход из аморфного состояния в кристаллическое сопровождается выделением скрытого тепла и изменением расстояний между атомами, т.е. указанный переход протекает в неравновесном нестационарном режиме. Известно, что в этих условиях могут наблюдаться, по крайней мере, два эффекта. Прежде всего при нагреве с конечной скоростью температура превращения снижается [2]. Далее, релаксация аморфной системы может протекать в бездиффузионном режиме за счет возбуждения динамического ближнего порядка [3]. Термин ,динамический означает, что данный ближний порядок возбуждается под действием внешних сил, амплитуда которых в рассматриваемом случае определяется градиентами температуры и величиной деформации. Динамический ближний порядок в свою очередь стимулирует установление дальнего порядка, сопровождающегося выделением скрытой теплоты превращения. Процессы возбуждения динамического ближнего порядка, дальнего порядка и теплопроводности связаны друг с другом. Но процесс теплопроводности является самым медленным, поэтому он определяет основные закономерности распространения волн кристаллизации. В связи с этим возникает задача об определении причины, условий, механизма зарождения и распространения температурного фронта при термоактивированном структурном превращении из метастабильного аморфного в устойчивое кристаллическое состояние при нагреве объемным тепловым источником [4]. Ее решению посвящена настоящая работа.

\section{1. Модель аморфно-кристаллического превращения}

Для исследования кинетики термоактивированного аморфно-кристаллического превращения, инициированного объемным тепловым источником [4], используем 
уравнение теплопроводности

$$
\frac{c \rho \partial T}{\partial t}=\lambda \frac{\partial^{2} T}{\partial x^{2}}+f+Q W(T)-\alpha\left(\frac{S}{V}\right)\left(T-T_{0}\right),
$$

где $c-$ коэффициент теплоемкости, $\rho-$ плотность среды, $T$ - температура образца, $T_{0}$ - температура окружающей образец среды (инертного газа $\operatorname{Ar}[1]), \lambda$ коэффициент теплопроводности, $f$ - объемная плотность мощности постоянного теплового источника, $\alpha-$ коэффициент теплоотвода, $Q=L \rho-$ скрытая удельная (на единицу объема) теплота превращения, $L$ - скрытая теплота структурного превращения, $W(T)$ - скорость превращения, $S, V$ - площадь поверхности и объем образца соответственно. Управляющий параметр $f$ можно исключить из (1), введя перенормированную температуру $\tilde{T}_{0}(f)$, линейно возрастающую с ростом параметра $f$,

$$
\tilde{T}_{0}(f)=T_{0}+(V f / S \alpha) .
$$

В дальнейшем знак „ “ будем опускать.

Объемная плотность мощности выделения скрытой теплоты превращения при аморфно-кристаллическом превращении подчиняется закону Аррениуса

$$
Q W(T, \eta)=Q W_{0}(T, \eta) \exp \left(-\frac{E}{k T}\right),
$$

где $\eta$ - удельное содержание аморфной фазы $(0 \leq$ $\leq \eta \leq 1), E-$ энергия активации структурного превращения, $W_{0}$ - коэффициент, слабо зависящий от температуры и определяемый частотой колебаний атомов. Из анализа экспериментальных данных для режима самопроизвольного превращения [1] следует, что критическая температура аморфной фазы перед фронтом $T_{0}^{*} \approx 573 \mathrm{~K}$, температура кристаллической фазы за фронтом $T_{b} \approx 723 \mathrm{~K}$, температура самопроизвольного (самоускоренного) превращения $T_{*} \approx 593 \mathrm{~K}$. Нагрев кристаллической фазы по отношению к аморфной (перепад температур) в результате превращения составляет $T_{b}-T_{0}^{*} \approx 150 \mathrm{~K}$, а предпереходный разогрев (медленный этап превращения) составляет $\theta_{0}^{*}=T_{*}-T_{0}^{*} \approx 20 \mathrm{~K}$. Период индукции (время предпереходного разогрева) составляет $\tau_{i} \approx 0.14 \mathrm{~s}$, а время зарождения температурного фронта (время самопроизвольного, самоускоренного, быстрого этапа превращения) составляет $\approx 0.025 \mathrm{~s}$. Эти экспериментальные данные позволяют вычислить скрытую теплоту превращения $L=c\left(T_{b}-T_{0}^{*}\right) \approx 80 \mathrm{~kJ} / \mathrm{kg}$ при $c=\left(c_{\mathrm{Ti}}+c_{\mathrm{Cu}}\right) / 2 \approx 0.5 \mathrm{~kJ} / \mathrm{kg} \cdot \mathrm{K}$, энергию теплового движения атома $k T_{0}^{*}=0.05 \mathrm{eV}$, характерную энергию активации процесса превращения $E=\frac{k T_{0}^{* 2}}{\theta_{0}^{*}} \approx 1.4 \mathrm{eV}$ (см. (5)), где $k$ - постоянная Больцмана. При таких значениях характерных параметров системы при самопроизвольном превращении для энергии активации, скрытой теплоты превращения выполняются неравенства

$$
\frac{E}{k T_{0}^{*}} \approx 28 \gg 1, \quad \theta_{0}^{*} \ll T^{*}, \quad \frac{T_{b}-T_{0}^{*}}{\theta_{0}^{*}} \approx 8 \gg 1 .
$$

Поэтому можно воспользоваться преобразованием Франка-Каменецкого для скорости превращения вблизи критической температуры $T=T_{0}^{*}$, равной начальной температуре аморфной фазы,

$$
W(T)=W_{0} e^{-E / k T}=W_{0}\left(T_{0}^{*}\right) e^{\frac{T-T_{0}^{*}}{\theta_{0}^{*}}}, \quad \theta_{0}^{*}=k T_{0}^{* 2} / E,
$$

где

$$
\begin{gathered}
W_{0}\left(T_{0}^{*}\right)=W_{0} e^{-E / k T_{0}^{*}}, \\
\frac{1}{T}=\frac{1}{\left(T_{0}^{*}+\left(T-T_{0}^{*}\right)\right)}=\frac{1}{T_{0}^{*}}\left(1-\frac{T-T_{0}^{*}}{T_{0}^{*}}\right) .
\end{gathered}
$$

Взаимосвязь температуры перед фронтом превращения $T_{0}$ за фронтом превращения $T_{b}$ и удельного содержания аморфной фазы $\eta$ определяется соотношениями

$$
\begin{gathered}
Q=\eta Q+c \rho\left(T-T_{0}\right)=c \rho\left(T_{b}-T_{0}\right), \\
T_{b}=T_{0}+\frac{Q}{c \rho}=T_{0}+\frac{L}{c},
\end{gathered}
$$

где $T_{b}$ - адиабатическая температура превращения. Используя (6), определим температуру $T_{m}$ и удельное содержание аморфной фазы $\eta_{m}$, при которой функция скорости превращения

$$
W(T, \eta)=W_{0}\left[\frac{T_{b}-T}{T_{b}-T_{0}}\right] \exp \left(-\frac{E}{k T}\right), \quad \eta=\left(\frac{T_{b}-T}{T_{b}-T_{0}}\right)
$$

имеет максимум,

$$
\begin{gathered}
\frac{T_{m}}{T_{b}}=1-\frac{k T_{b}}{E} \cong 1, \\
\eta_{m}=\left(\frac{k T_{b}^{2}}{E}\right)\left[T_{b}-T_{0}\right]^{-1} \approx 0.2 \ll 1 .
\end{gathered}
$$

При получении (8) учитывалось (4). Большая величина энергии активации показывает, что имеется потенциальный барьер, который необходимо преодолеть для протекания превращения, поэтому скорость превращения при низких температурах мала. Однако при увеличении температуры скорость превращения быстро растет по закону Аррениуса. При этом увеличение скорости превращения сопровождается увеличением выделения тепла и следовательно возрастанием температуры. Таким образом, реализуется самоускоряющийся процесс, который будет происходить до тех пор, пока все аморфное вещество не превратится в кристаллическое. Падение скорости перехода будет иметь место только при низком удельном содержании аморфной фазы. Температура $T_{m}$, при которой достигается максимум скорости превращения, близка к адиабатической температуре превращения, а удельное содержания аморфной фазы $\eta_{m}$ мало. Резкое возрастание скорости превращения как функции температуры по закону Аррениуса и затем ее резкое падение из-за уменьшения удельного содержания аморфной фазы делает ее похожей на дельта-функцию, поэтому приближенно можно считать, что превращение 
происходит в основном только в небольшой окрестности ниже адиабатической температуры. Время от начала процесса $T_{0}^{*}$ до достижения температуры $T_{*}$ называется периодом индукции. В течение периода индукции температура и удельное содержание аморфной фазы меняются слабо, а затем начинается быстрый самоускоряющийся этап превращения, который заканчивается нагревом до адиабатической температуры и полным превращением аморфной фазы.

\section{2. Определение характеристик самопроизвольного структурного превращения}

\section{1. Температура самопроизвольного структурного превращения}

Рассмотрим однородные стационарные решения (1)

$$
Q W_{0} \exp \left(-\frac{E}{k T_{*}}\right)=\left(\frac{\alpha S}{V}\right)\left(T_{*}-T_{0}\right) .
$$

Соответствующая стационарному состоянию температура будет точкой пересечения кривых теплоотвода и тепловыделения. При низких значениях $T_{0}(f)$ имеются две точки пересечения кривых. Первая близка по значению к $T_{0}$, а вторая заметно выше. Первое стационарное состояние аморфной среды с температурой $T_{a}$ будет устойчивым, поскольку дальнейшее повышение температуры сопровождается превышением теплоотвода над тепловыделением. Вторая точка пересечения кривых лежит в области более высоких температур, и тепловой режим системы будет неустойчивым. При увеличении управляющего параметра $f$ прямая $T_{0}(f)$, характеризующая теплоотвод, смещается вправо, а температура, характеризующая устойчивое состояние аморфной среды $T_{a}$, непрерывно и плавно возрастает. При некоторой критической температуре $T_{0}\left(f_{*}\right)=T_{0}^{*}$ кривые теплоотвода и тепловыделения только соприкоснутся. Это означает равенство величин теплоотвода и тепловыделения, а также их производных в точке $T_{*}\left(f_{*}\right)=T_{*}$. Эта точка характеризует граничные условия существования стационарного режима. При незначительном превышении $T_{0}(f)$ по сравнению с $T_{0}\left(f_{*}\right)$ тепловыделение будет превышать теплоотвод, и произойдет резкое увеличении температуры среды. Термоактивированное явление, заключающееся в переходе от медленно протекающего превращения, сопровождающегося незначительным разогревом, к быстрому самоускоряющемуся превращению, называют самопроизвольным (самоускоренным) аморфно-кристаллическим превращением. В научной литературе известен его аналог для горючих газов, называющийся „тепловым взрывом“6 $[5,6]$.

В точке касания кривых теплоотвода и тепловыделения, где удельное содержание аморфной фазы близко к единице, имеют место равенства

$$
\begin{gathered}
Q W_{0} \exp \left(-\frac{E}{k T_{*}}\right)=\left(\frac{\alpha S}{V}\right)\left(T_{*}-T_{0}^{*}\right), \\
Q W_{0}\left(\frac{E}{k T_{*}^{2}}\right) \exp \left(-\frac{E}{k T_{*}}\right)=\left(\frac{\alpha S}{V}\right) .
\end{gathered}
$$

Разделив правые и левые части уравнений (10), (11) получим

$$
T_{*}=\left(\frac{E}{2 k T}\right)\left[1 \mp \sqrt{1-\frac{4 k T_{0}^{*}}{E}}\right] .
$$

Физической температуре самопроизвольного структурного превращения $T_{*}$ в формуле (12) соответствует знак минус перед значением квадратного корня. Поскольку энергия активации структурного превращения $E$ много больше тепловой энергии атома $k T_{0}^{*}$, то подкоренное выражение (12) можно заменить разложением в ряд по этому малому параметру и ограничиться тремя первыми членами разложения. Тогда

$$
T_{*}=T_{0}^{*}+\theta_{0}^{*} .
$$

Величину $\theta_{0}^{*} \approx 20 \mathrm{~K}$ называют предпереходным разогревом, который протекает за период индукции. После него самопроизвольное превращение протекает самоускоренно и быстро. После подстановки выражения (12) в формулу (10) находится трансцендентное уравнение на критическую температуру

$$
\left(\frac{\alpha S}{V}\right)\left(\frac{E}{k T_{0}^{* 2}}\right)\left(Q W_{0}\right)^{-1} \exp \left(\frac{E}{k T_{0}^{*}}\right)=e .
$$

Численное решение этого уравнения дает $T_{0}^{*} \approx 573 \mathrm{~K}$. Используя экспериментальные данные для периода индукции (около $0.14 \mathrm{~s}$ ) [1] и формулы (13), (14), получим оценку скорости превращения при $T=T_{0}^{*}, T=T_{*}$ :

$$
W\left(T_{0}^{*}\right) \approx 1.3 \mathrm{~s}^{-1}, \quad W\left(T_{*}\right) \approx 3.6 \mathrm{~s}^{-1} .
$$

В случае самопроизвольного структурного превращения подводимое к системе тепло распределяется равномерно по всему объему системы. При достижении управляющим параметром значения $f_{*}$ температура аморфной фазы достигает значения $T_{0}^{*}$. В течение периода индукции происходит медленный самопроизвольный предпереходный разогрев локальной области близко к центру системы, где потери тепла минимальны. Когда температура этой локальной области достигает температуры самопроизвольного структурного превращения $T_{*}$, начинается быстрый, самоускоряющийся этап превращения и формируются температурные фронты. После этого возникшие фронты превращения распространяются по всему объему системы. Температура самопроизвольного структурного превращения $T_{*}$ зависит от критической температуры $T_{0}^{*}$, теплофизических характеристик вещества и находится в области высоких температур. Важно, что $T_{*}$ не является физической константой, связанной только с параметрами скорости превращения $E, W_{0}$, а зависит (через параметр $T_{0}^{*}$ ) от формы и размеров системы, коэффициента теплоотвода. 


\section{2. Время индукции структурного самопроизвольного превращения}

Важнейшей характеристикой самопроизвольного превращения является период индукции. Найдем вначале критические условия самопроизвольного превращения, т.е. значения параметров системы на границе между двумя возможными режимами протекания процесса стационарным медленным превращением и самопроизвольным быстрым превращением, а также время индукции самопроизвольного структурного превращения. Подставляя в уравнение (1) формулы (2), (3), применяя преобразования Франка-Каменецкого (5) вблизи температуры $T=T_{0}^{*}$ и переходя к безразмерным переменным

$$
\begin{gathered}
\tau=\frac{t}{t_{i a}}, \quad t_{i a}=\frac{\rho c \theta_{0}^{*} e^{\frac{E}{k T_{0}^{*}}}}{Q W_{0}}, \\
\varphi=\frac{T-T_{0}^{*}}{\theta_{0}^{*}}, \quad t_{\alpha}=\frac{\rho c V}{\alpha S}, \quad \gamma=\frac{t_{i a}}{t_{a}},
\end{gathered}
$$

получим уравнение

$$
\frac{\partial \varphi}{\partial \tau}=\exp \varphi-\gamma \varphi
$$

Здесь параметр $\gamma$ равен отношению адиабатического периода индукции $t_{i a}$ к характерному времени теплоотвода $t_{\alpha}$. Параметр $\gamma$ зависит от объема и площади поверхности системы, теплотворной способности и теплопроводности системы, ее кинетических характеристик и температуры внешней среды.

Условия перехода от стационарного режима медленного превращения к самопроизвольному быстрому превращению могут быть записаны как условие касания кривых $\exp (\varphi)$ и $\gamma \varphi$ :

$$
\exp (\varphi)=\gamma \varphi, \quad \frac{d \exp (\varphi)}{d \varphi}=\frac{d \gamma \varphi}{d \varphi}
$$

Отсюда следует, что условия самопроизвольного превращения имеют вид

$$
\gamma=e, \quad \varphi=1
$$

Второе условие $\left(T_{*}-T_{0}^{*}=\theta_{0}^{*}\right)$ определяет максимальный предпереходный разогрев системы, который совпадает с (13) при условии $\frac{E}{k T_{0}^{*}} \ll 1$. Звездочкой обозначаются величины на пределе самопроизвольного превращения. Для превращения [1] $E \approx 1.4 \mathrm{eV}, T_{0}^{*} \approx 573 \mathrm{~K}$ характеристический интервал $\theta_{0}^{*} \approx 20 \mathrm{~K}$.

Общее выражение для периода индукции находится путем интегрирования уравнения (17)

$$
\tau_{i}=\int_{0}^{\infty} d \varphi[\exp (\varphi)-\gamma \varphi]^{-1}
$$

Если система находится достаточно „глубоко“ в области самопроизвольного превращения, то теплопотерями можно пренебречь, и период становится равным адиабатическому $t_{i a}$.

Сделаем качественные выводы о величине периода индукции и временном ходе кинетических кривых $T(t)$. При $T<T_{0}^{*}$ превращение выходит на медленный стационарный режим, а соответствующая кинетическая кривая $T(t)$ имеет плато с температурой $T_{a}$. При $T>T_{0}^{*}$ происходит быстрое самопроизвольное превращение, а соответствующая кинетическая кривая $T(t)$ резко изменяет свой характер. Первоначальный рост кривой замедляется для моментов времени, соответствующих области максимального сближения кривых теплоприхода и теплоотвода, затем температура быстро возрастает. Период индукции в этом случае практически равен адиабатическому $t_{i a}$.

При начальной температуре $T \geq T_{0}^{*}$, но близкой к $T_{0}^{*}$ период индукции сильно затягивается. Это связано с тем, что в этом случае система долго находится при температурах, отвечающих максимальному сближению кривых теплоприхода и теплоотвода и весьма малой величине производной $d T / d t$. При вычислении периода индукции самопроизвольного превращения для таких кинетических кривых уже нельзя пренебрегать теплоотводом. Приближенное значение периода индукции по (17) вблизи предела самопроизвольного превращения можно найти, считая, что значительную часть времени система затрачивает на прохождение узкой „щели“ между линиями теплоприхода и теплоотвода, т.е. система находится вблизи точки со значениями параметров

$$
\gamma=(1-\vartheta) e, \quad \varphi=1+\mu,
$$

где $\vartheta, \mu \ll 1$. Подставляя (21) в (20) и пренебрегая малыми членами высших порядков, находим

$$
\tau_{i} \approx \sqrt{e /(e-\gamma)}
$$

Отсюда следует, что на пределе самопроизвольного превращения $\gamma \rightarrow e$ период индукции стремится к бесконечности. Период индукции при приближении к критической точке $T_{0}^{*}$ сверху при фиксированных значениях остальных параметрах стремится к бесконечности по закону $\tau_{i} \approx\left(T_{0}-T_{0}^{*}\right)^{-1}$.

Оценка периода индукции для эксперимента [1] дает величину $t_{i \exp } \approx 0.14 \mathrm{~s}$. Используя (16) и характерные значения параметров процесса, находим адиабатический период индукции системы $t_{i a} \approx 0.1 \mathrm{~s}$. Видно, что экспериментальный период индукции в 1.4 раза больше адиабатического периода индукции. Следовательно, в эксперименте [1] система находится на $10 \mathrm{~K}$ выше критической точки превращения $T_{0}^{*}$, а адиабатический период индукции в $(e / 2)$ раза больше характерного времени теплоотвода $t_{\alpha}$.

\section{3. Скорость распространения фронта структурного превращения}

Экспериментальные данные и теоретические расчеты свидетельствуют о том, что при распространении фрон- 
та превращение в каждый момент времени идет в сравнительно тонком слое - зоне превращения, составляющей 4-6 межатомных расстояний системы. Разогрев вещества происходит в непосредственной близости к зоне превращения, также в тонком слое - зоне подогрева, которая значительно больше зоны превращения. Поэтому среду, в которой протекает превращение, в первом приближении можно представлять в виде двух областей, разделенных поверхностью превращения. В одной из областей превращение протекает, а во второй - нет. Толщиной поверхности можно пренебречь, и рассматривать ее как геометрическую поверхность, движущуюся относительно системы с известной скоростью $u$.

Величина скорости распространения превращения определяется его кинетикой и атомными процессами переноса тепла внутри фронта превращения, где существует большой градиент температуры. При анализе явлений, происходящих у поверхности превращения, удобно выбирать локальную систему координат, связанную с фронтом превращения. Превращение внутри температурного фронта протекает неоднородно из-за резкой зависимости скорости превращения от температуры. Оно, главным образом, сосредоточено в части температурного фронта, прилегающего к разогретой, превращенной части системы. Там, где температура ниже, превращение протекает со значительно меньшей скоростью. Поэтому его можно вообще не учитывать, а рассматривать только перенос тепла. Определим порядок величины зоны прогрева.

Уравнение теплопроводности (1) для не превращенной части образца в системе координат, связанной с фронтом превращения, вместе с граничными условиями при пренебрежении членами, связанными с теплоотводом и тепловыделением, приводится к виду

$$
\frac{c \rho u d T}{d x}=\frac{\lambda d^{2} T}{d x^{2}}, \quad T(-\infty)=T_{0}, \quad T(0)=T_{b} .
$$

Решение уравнения (23), применимое к области $x<0$, в которой тепловыделение отсутствует, имеет вид

$$
T(x)=T_{0}+\left(T_{b}-T_{0}\right) \exp \left[-\left(\frac{u}{a}\right)|x|\right],
$$

где $T_{0}\left(T_{b}\right)$ - температура до температурного фронта (после фронта) превращения, $u$ - скорость фронта, $a$ - коэффициент температуропроводности. В качестве масштаба ширины зоны подогрева $l$ можно принять расстояние, на котором разогрев уменьшается в $e$ раз. Из (23) получаем

$$
l=a / u=\lambda / \rho c u
$$

Для $\mathrm{Cu}_{50} \mathrm{Ti}_{50}$ с коэффициентом температуропроводности $a \approx 4 \cdot 10^{-6} \mathrm{~m}^{2} / \mathrm{s}$ и скоростью фронта $u \approx 0.012 \mathrm{~m} / \mathrm{s}$, находим $l \approx 0.4 \cdot 10^{-3} \mathrm{~m}$. По этой причине вкладом изменения конвективного потока тепла в зоне превращения можно пренебречь по сравнению с вкладом теплопроводности (в зоне превращения перепад температур мал). Таким образом, уравнение (1) в зоне превращения примет вид

$$
\lambda \frac{\partial^{2} T}{\partial x^{2}}+Q W(T)=0
$$

Уравнение (26) легко интегрируется. Количество тепла, выделяемое при превращении в единицу времени и отводящееся теплопроводностью через единицу площади поверхности, равно произведению скрытой теплоты превращения и скорости распространения фронта

$$
\left[2 \lambda Q \int_{T}^{T_{b}} W(T) d T\right]^{1 / 2}=Q u
$$

Таким образом,

$$
u=\sqrt{\left(\frac{2 \lambda}{Q}\right) \int_{T}^{T_{b}} W(T) d T} .
$$

Видно, что скорость превращения зависит от интеграла функции тепловыделения. Поэтому она связана с кинетикой превращения, с энергией активации, с другими кинетическими и тепловыми характеристиками среды. Когда энергия активации превращения много больше энергии теплового движения атомов, можно использовать разложение Франка-Каменецкого для скорости $W(T)$. Тогда (28) примет вид

$$
u=\sqrt{\frac{2 \lambda}{Q} W\left(T_{b}\right) \theta_{b}}=\mathrm{const} \sqrt{a / t_{r}}, \quad \text { const }=\sqrt{\frac{2 \theta_{b}}{T_{b}-T_{0}}} .
$$

Здесь время самоускоряющегося этапа превращения $t_{r} \approx 0.025 \mathrm{~s}$ - это время, за которое при максимальной скорости тепловыделения выделится все тепло в зоне превращения. Заметим, что соотношение (29) вытекает также из соображений анализа размерности. Подставляя характерные значения параметров системы для режима инициированного превращения [1] $T_{b}=673 \mathrm{~K}, T_{0}=523 \mathrm{~K}, \theta_{b}=27 \mathrm{~K}, W(673 \mathrm{~K}) \approx 96 \mathrm{~s}^{-1}$ в формулу (28), получим

$$
u(T=673 \mathrm{~K})=12 \mathrm{~mm} / \mathrm{s} .
$$

В зоне превращения протекает быстрый переход в узком интервале температур, примыкающем снизу к температуре $T_{b}$. Тепло, выделяющееся в превращении, расходуется в основном на подогрев не превращающейся части вещества от начальной температуры до температуры зоны превращения. В узкой зоне превращения производная температуры изменяется от конечного значения со стороны не превращающейся части вещества (из зоны превращения тепло теплопроводностью предается в зону прогрева) до нуля со стороны превращенной части вещества, где достигается тепловое равновесие. Быстрое изменение производной температуры на коротком пространственном промежутке означает большую величину вклада теплопроводности. Скорость распространения 
фронта превращения в явном виде обратно пропорциональна тепловому эффекту $Q$. Однако если учесть, что адиабатическая температура превращения $T_{b}$ также растет с увеличением $Q$, то скорость распространения фронта в действительности возрастает с увеличением теплового эффекта. При постоянном тепловом эффекте скорость быстро экспоненциальным образом (за счет скорости превращения) растет с ростом адиабатической температуры превращения $T_{b}$.

\section{3. Основные выводы и заключение}

Причиной возникновения термоактивированного аморфно-кристаллического превращения является накопление тепла в системе при нагревании тепловым источником. Система испытывает превращение только при определенных условиях: среда должна быть структурно неустойчивой, $\mathrm{a}$ превращение должно протекать с выделением тепла, быть экзотермическим, причем скорость превращения должна возрастать с увеличением температуры.

Режим возникновения и распространения превращения связан с атомными процессами теплопроводности, а механизм явления имеет тепловой характер. При тепловом механизме возникновения и распространения фронта превращения тепло, выделившееся при превращении, теплопроводностью передается на соседние участки не превращенного вещества, нагревает их и инициирует самоускоряющийся переход.

В случае самопроизвольного структурного превращения подводимое к системе тепло распределяется равномерно по всему объему системы. Температура самопроизвольного структурного превращения $T_{*}$ зависит от критической температуры $T_{0}^{*}$, теплофизических характеристик вещества и находится в области высоких температур. Важно, что $T_{*}$ не является физической константой, связанной только с параметрами скорости превращения $E, W_{0}$, а зависит (через параметр $T_{0}^{*}$ ) от формы и размеров системы, коэффициента теплоотвода.

На пределе самопроизвольного превращения период индукции стремится к бесконечности. Если приближаться сверху к критической точке $T_{0}^{*}$ при фиксированных остальных параметрах, то период индукции стремится к бесконечности по закону $\tau_{i} \approx\left(T_{0}-T_{0}^{*}\right)^{-1}$. Если система находится достаточно „глубоко“ в области самопроизвольного превращения, то теплопотерями можно пренебречь, и период становится равным адиабатическому $t_{i a}$.

Величина скорости распространения превращения определяется его кинетикой и атомными процессами переноса тепла внутри температурного фронта, где существует большой градиент температуры. Отличительной особенностью кинетики превращения в температурном фронте волны превращения является освобождение превращения от всех задерживающих стадий. В то время как на стадии самопроизвольного превращения накопление в системе тепла задерживает развитие процесса, во фронте волны эти медленные процессы отсутствуют: в интервале температур, в котором нагревание среды за счет тепловыделения шло бы медленно, тепловой поток из соседнего слоя вызывает быстрый подъем температуры.

Таким образом, предлагаемая модель кинетики позволяет сформулировать причину, условия, механизм термоактивированного аморфно-кристаллического превращения в $\mathrm{Ti}_{50} \mathrm{Cu}_{50}$, инициированного объемным тепловым источником, количественно и качественно описывает результаты и закономерности распространения температурного фронта, полученные экспериментально.

Работа выполнена по Программе фундаментальных исследований государственных академий наук на 2013-2020 гг. (проект 23.1.2). The research was supported in part by Marie Curie International Research Staff Exchange Scheme Fellowship within the 7th European Community Framework Programme (Grant Agreement Number 612552).

\section{Список литературы}

[1] Рогачев А.С., Вадченко С.Г., Щукин А.С., Ковалев И.Д., Аронин А.С. // Письма в ЖЭТФ. 2016. Т. 104. Вып. 10. C. $740-744$.

[2] Шмытько И.М., Афонникова Н.С., Дорохова Н.А. // ФТТ. 1998. T. 40. № 12. C. 2217-2220.

[3] Егорушкин В.Е., Мельникова Н.В. // ЖЭТФ. 1993. Т. 103. Вып. 2. С. 214-226.

[4] Слядников Е.Е., Турчановский И.Ю. // Изв. вузов. Физика. 2016. Т. 59. № 9. С. 125-133.

[5] Семенов Н.Н. // УФН. 1940. Т. 23. Вып. 3. С. 251-292.

[6] Зельдович Я.Б. Избранные труды. Химическая физика и гидродинамика. М.: Наука, 1984. 374 с. 